\title{
DETERMINAÇÃO EXPERIMENTAL E MODELAGEM TERMODINÂMICA DA ADSORÇÃO DE DIESEL UTILIZANDO BAGAÇO DE CANA COMO MATERIAL ADSORVENTE
}

\author{
T. B. A. OLIVÉRIO ${ }^{1}$, H.O. N. ALTINO ${ }^{2}$ e S. ARVELOS ${ }^{1}$ \\ ${ }^{1}$ Centro Universitário de Patos de Minas, Departamento de Engenharia Química \\ ${ }^{2}$ Universidade Federal de São Carlos, Departamento de Engenharia Química \\ E-mail para contato: thauaneaoliverio@gmail.com
}

\begin{abstract}
RESUMO - O uso de processos adsortivos para a remoção de derivados de petróleo existentes em efluentes tem se destacado no meio de pesquisa por se tratar de um método de baixo custo. $\mathrm{O}$ presente trabalho teve como objetivo estudar a remoção de diesel de uma solução aquosa utilizando bagaço de cana e estudar a termodinâmica da adsorção. Para isto, o mesmo realizou ensaios experimentais visando avaliar a cinética e o equilíbrio termodinâmico. Os resultados obtidos mostraram curvas cinéticas de remoção com curto tempo de saturação. Quanto ao equilíbrio, foi possível concluir que para as altas concentrações de diesel, o tratamento ácido provê ao bagaço de cana maior capacidade adsortiva que o bagaço tratado sob pressão. $\mathrm{Na}$ modelagem do equilíbrio, o modelo bi-Langmuir foi o modelo que representou melhor os sistemas estudados.
\end{abstract}

\section{INTRODUÇÃO}

A poluição das águas por derivados do petróleo é um dos grandes problemas da sociedade atual, pois contamina as fontes de água potável, envenena os animais e as plantações. Como forma de tratamento desse tipo de efluente e/ou mananciais contaminados em derramamentos, os processos adsortivos se destacam. Dentro da pesquisa nesta área de processos, a busca por matrizes porosas de baixo custo têm sido aprofundada (Rubio et al., 2003).

O bagaço de cana é um material de produção abundante no Brasil. Diversos autores já investigaram a sua influência na remoção de componentes orgânicos de águas residuais (Brandão et al., 2010). O processo de adsorção é uma forma de incorporar valor ao bagaço de cana, pois este seria um produto que seria descartado pela indústria do açúcar e do álcool. Além disso, o bagaço de cana com material residual da adsorção teria uma aplicação prática industrial concomitante que enriquecimento energético do bagaço para sua posterior utilização como combustível em caldeiras (Rowell; Keany, 1991).

O presente trabalho objetiva estudar a adsorção de diesel em bagaço de cana pré-tratado com ácido. O tratamento ácido será utilizado para modificação da estrutura e disponibilização dos sítios ativos. Além disso, deseja-se estudar a cinética de adsorção e avaliar a viabilidade de se adaptar uma isoterma classicamente empregada para adsorção de fase fluida gasosa para a fase fluida líquida. 


\section{REFERENCIAL TEÓRICO}

\subsection{Cinética de Adsorção}

Objetivando estudar a cinética de adsorção, modelos devem ser estudados de forma a avaliar o melhor ajuste aos dados experimentais. Um modelo bastante utilizado na literatura é o modelo de Lagergren, que assume que a cinética de adsorção é de primeira ordem (Iljina; Baltakys; Eisinas, 2015). Este modelo, na forma integrada, apresenta-se da seguinte forma:

$$
\log \left(q_{e}-q\right)=\log \left(q_{e}\right)-\frac{k t}{2,303}
$$

na qual $q_{e}$ e $q$ representam a capacidade de adsorção no equilíbrio e em um dado tempo $t$, respectivamente, e $k$ é a constante da taxa para a adsorção de primeira ordem $\left(\mathrm{min}^{-1}\right)$.

Caso se assuma um modelo de segunda ordem para representação da cinética, a equação, na forma integrada, se expressa como na Equação 2, onde $k_{2}$ é a constante da taxa (g.mL ${ }^{-1} \cdot \mathrm{min}^{-1}$ ) (Qiu et al., 2009). Esta equação tem sido utilizada com sucesso para descrever a adsorção de óleos e substâncias orgânicas de soluções aquosas (Chen et al., 2008).

$$
\frac{1}{q}=\frac{1}{k_{2} q_{e}^{2}}+\frac{1}{q_{e}} t
$$

\subsection{Equilíbrio de Adsorção}

O modelo de Langmuir (ML) já é amplamente utilizado para adsorção em líquidos na literatura. $\mathrm{O}$ modelo pode ser representado para adsorção de líquidos, substituindo a pressão parcial pela concentração do adsorvato. A Equação 3 apresenta o modelo em questão:

$$
q=q_{\max } \frac{K C}{1+K C}
$$

onde $K$ representa a constante de Henry, $C$ a concentração no equilíbrio e $q_{\max }$ representa a quantidade máxima adsorvida referente à formação da monocamada. Adsorventes provenientes de biomassas, como o bagaço de cana são constituídos de diversos compostos tais como hemicelulose, lignina e celulose (Macrelli et al., 2012). Logo, espera-se que exista uma diferença de energia entre os sítios de adsorção. Um modelo para superfícies nãohomogêneas é o modelo bi-Langmuir (MBL) apresentado abaixo:

$$
q=q_{1, \max } \frac{K_{1} C}{1+K_{1} C}+q_{2, \max } \frac{K_{2} C}{1+K_{2} C}
$$

neste modelo, a superfície é divida em duas regiões homogêneas de adsorção (patches), sendo a capacidade máxima adsorvida dada termos da soma de $q_{1, \max }$ e $q_{2 \text {,max }}$ que representam a capacidade de saturação da cada região. Cada patch tem sua própria constante de equilíbrio $\left(K_{1}\right.$ e $\left.K_{2}\right)$. 


\section{MATERIAL E MÉTODOS}

\subsection{Parte Experimental}

Para o estudo da adsorção de diesel o bagaço de cana foi disponibilizado por uma indústria de cachaçaria, onde foi devidamente armazenado e levado ao laboratório de engenharia química do UNIPAM. O material foi lavado com água destilada até a neutralização do $\mathrm{pH}$ e a clarificação da água de lavagem. $\mathrm{O}$ bagaço foi submetido a um tratamento com $1,2 \mathrm{~mol} / \mathrm{L}$ de ácido cítrico à base de água morna e em seguida o material foi seco em estufa a $60^{\circ} \mathrm{C}$ durante 12 horas (Rodrigues et al., 2006). O material foi levado à moagem em liquidificador industrial para obter-se granulometria inferior a $1 \mathrm{~mm}$ e após o processo de tratamento foi armazenado em condições controladas de umidade e temperatura.

A cinética de adsorção foi feita utilizando-se a metodologia proposta por Vasconcelos e Romanielo (2011), a partir do fracionamento do diesel. A repartição foi feita em béqueres de $600 \mathrm{~mL}$ onde foram empregadas à mesma quantidade de água $(120 \mathrm{~mL}), 3 \mathrm{~g}$ do bagaço de cana armazenado e proporções variáveis de diesel. Estes recipientes que foram submetidos à agitação mecanizada, à temperatura ambiente. Em seguida, foram registrados tempos distintos de agitação (entre 10 e 60 minutos). A seguir, as amostras foram submetidas a uma filtração a vácuo em filtro de Buchner. O filtrado colocado em uma proveta para decantação das fases (água e diesel). Pela leitura da diferença de volume entre os líquidos antes e após os experimentos refere-se à quantidade adsorvida pelo bagaço de cana. Os experimentos foram realizados em duplicata para diminuir o grau de incerteza. As quantidades variáveis de diesel adicionadas em cada béquer estão apresentadas na Tabela 1.

Além da cinética, uma isoterma de equilíbrio foi obtida experimentalmente. Novas repartições de diesel foram realizadas em solução na presença do adsorvente $(120 \mathrm{~mL}$ de água, $3 \mathrm{~g}$ de bagaço e quantidades variáveis de diesel). Os volumes de diesel adicionados aos béqueres de $600 \mathrm{~mL}$ foram 42, 36, 30, 24, 18 e $12 \mathrm{~mL}$. O tempo de contato estabelecido foi de 120 minutos, tempo suficiente de obtenção do equilíbrio (El-Gendy; Nassar, 2015).

Tabela 1 - Razões contaminante/adsorvente utilizadas nos ensaios de cinética de adsorção

\begin{tabular}{ccc}
\hline Volume de Diesel $(\mathrm{mL})$ & Quantidade de adsorvente $(\mathrm{g})$ & Proporção diesel/adsorvente $(\mathrm{mL} / \mathrm{g})$ \\
\hline 18 & 3,0 & 6,0 \\
24 & 3,0 & 8,0 \\
30 & 3,0 & 10,0 \\
36 & 3,0 & 12,0 \\
\hline
\end{tabular}

Fonte: Autoria própria (2017)

\subsection{Modelagem Termodinâmica}

Para estudo da cinética, os dados experimentais obtidos neste trabalho foram utilizados na regressão dos parâmetros dos modelos de primeira e segunda ordem. As curvas cinéticas obtidas foram comparadas com os resultados de Vasconcelos e Romanielo (2011) que admitiram as curvas cinéticas de adsorção de diesel com bagaço tratado sob pressão.

Os modelos de Langmuir e bi-Langmuir foram utilizados para modelagem da isoterma de equilíbrio obtida. Além da isoterma obtida neste trabalho, foram consultados dados na 
literatura disponível referentes à adsorção de hidrocarbonetos à temperatura ambiente para complementação do estudo. O referencial bibliográfico dos dados consultados está apresentado na Tabela 3. Os trabalhos citados nesta Tabela abordam a adsorção em bagaço in natura, exceto para o Diesel ${ }^{\mathrm{b}}$, que foi obtido em bagaço tratado sob pressão. O software utilizado para regressão dos parâmetros foi o Statistica ${ }^{\circledR}$ 7.0, tendo por base o método dos mínimos quadrados.

\section{RESULTADOS E DISCUSSÃO}

A Figura 1(a) apresenta a influência do tempo de contato entre as fases sobre a quantidade de diesel adsorvido, para as quatro diferentes concentrações iniciais de diesel na solução. Pode ser notado que, a cinética é claramente influenciada pela razão inicial de diesel/adsorvente. Quanto maior o volume inicial de diesel, maior a quantidade adsorvida no equilíbrio. As quatro curvas cinéticas avaliadas foram comparadas com as obtidas em Vasconcelos e Romanielo (2011) para a adsorção em bagaço tratado sob pressão. Foi possível notar que o tempo para alcançar o equilíbrio é menor quando o bagaço é tratado pelo tratamento ácido o que pode ser explicado pela maior acessibilidade aos sítios ativos, provocada pelo provável aumento da área superficial. Ambos os trabalhos apresentaram concentrações do equilíbrio bastante próximas. Assim, o bagaço tratado pela técnica com ácido apresenta desempenhos ligeiramente melhores que o tratamento por pressão. Mas, no geral, percebe-se que ambas as biomassas tratadas apresentam capacidade de remoção equivalente uma vez que a incerteza na medida máxima obtida neste trabalho é de $2,81 \%$. A Figura 1(b) e 1(c) apresenta a comparação das cinéticas para duas diferentes razões de diesel/adsorvente.

Figura 1 - Cinéticas de adsorção de diesel em bagaço de cana. (a) Cinéticas obtidas neste trabalho. (b) Comparação entre as curvas obtidas neste trabalho e a literatura

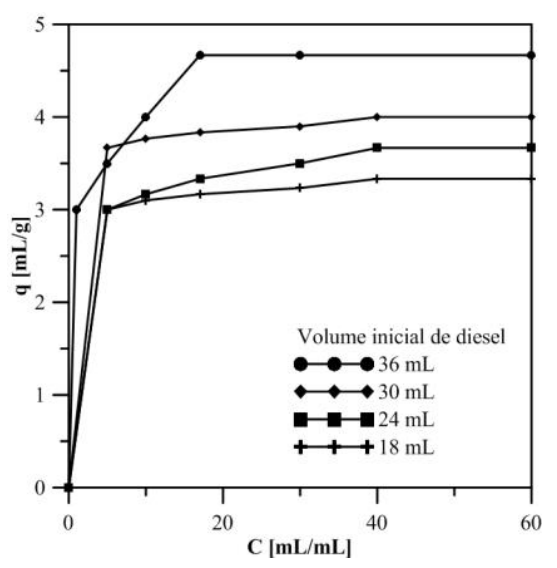

(a)

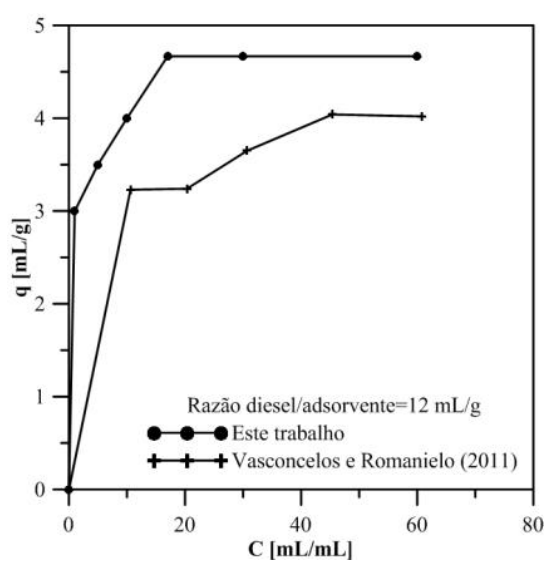

(b)

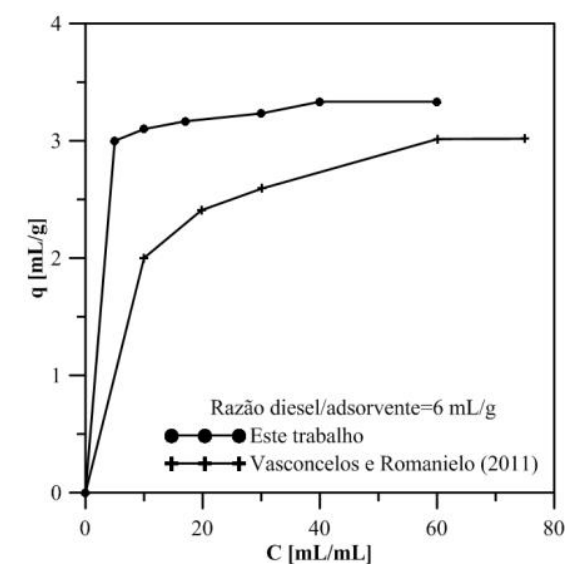

(c)

Os dados experimentais obtidos neste trabalho foram modelados frente às cinéticas de primeira e segunda ordem. Foi possível notar que a cinética é de segunda ordem, devido aos maiores coeficientes de correlação. Os parâmetros obtidos através de regressão são apresentados na Tabela 2. 
Tabela 2 - Parâmetros obtidos para os modelos de primeira e segunda ordem

\begin{tabular}{ccccccc}
\hline $\begin{array}{c}\text { Volume } \\
\text { inicial }[\mathrm{mL}]\end{array}$ & \multicolumn{3}{c}{ Primeira ordem } & \multicolumn{3}{c}{ Segunda ordem } \\
\cline { 2 - 7 } & $\mathrm{q}_{\mathrm{e}}[\mathrm{mL} / \mathrm{g}]$ & $k_{1}\left[\mathrm{~min}^{-1}\right]$ & $\mathrm{R}^{2}$ & $\mathrm{q}_{\mathrm{e}}[\mathrm{mL} / \mathrm{g}]$ & $k_{2}\left[\mathrm{~g} \cdot \mathrm{mL}^{-1} \cdot \mathrm{min}^{-1}\right]$ & $R^{2}$ \\
\hline 36 & 1,8806 & 0,1022 & 0,9960 & 3,04599 & 0,1161 & 0,9544 \\
24 & 0,3921 & 0,0467 & 0,9838 & 0,6768 & 0,2803 & 0,9969 \\
18 & 0,8707 & 0,0555 & 0,9995 & 3,5473 & 0,1834 & 0,9745 \\
& 0,3921 & 0,0467 & 0,9838 & 0,6768 & 0,2803 & 0,9969 \\
\hline
\end{tabular}

Fonte: Autoria própria (2017)

Quanto à modelagem do equilíbrio, os parâmetros referentes aos modelos de Langmuir e Bi-Langmuir são apresentados na Tabela 3. O modelo Bi-Langmuir apresentou melhores coeficientes de correlação tendo em vista da adição de 2 novos parâmetros para representação da heterogeneidade do sólido. A Figura 2 apresenta a isoterma de equilíbrio obtida neste trabalho e a obtida por Romanielo et al. (2008) para a adsorção de diesel. Pode ser notado que apesar da melhor correlação do MBL frente ao ML, ambos os modelos representam quantitativamente as isotermas em questão.

Tabela 3 - Parâmetros obtidos para os modelos ML e MBL.

\begin{tabular}{|c|c|c|c|c|c|c|c|c|c|}
\hline \multirow[b]{2}{*}{ Adsorvato } & \multirow[b]{2}{*}{ Referência } & \multicolumn{3}{|c|}{ Parâmetros ML } & \multicolumn{5}{|c|}{ Parâmetros MBL } \\
\hline & & $\begin{array}{c}K \\
{[\mathrm{~mL} / \mathrm{mL}]}\end{array}$ & $\begin{array}{c}q_{\max } \\
{[\mathrm{mL} / \mathrm{g}]}\end{array}$ & $\mathrm{R}^{2}$ & $\begin{array}{c}K_{I} \\
{[\mathrm{~mL} / \mathrm{mL}]}\end{array}$ & $\begin{array}{c}K_{2} \\
{[\mathrm{~mL} / \mathrm{mL}]}\end{array}$ & $\begin{array}{c}q_{1, \max } \\
{[\mathrm{mL} / \mathrm{g}]}\end{array}$ & $\begin{array}{c}q_{1, \max } \\
{[\mathrm{mL} / \mathrm{g}]}\end{array}$ & $\mathrm{R}^{2}$ \\
\hline Diesel $^{\mathrm{a}}$ & Este trabalho & 22,48 & 5,59 & 0,96 & 1,05 & 129,34 & 12,39 & 1,05 & 0,98 \\
\hline Diesel & Vasconcelos; & 273,49 & 3,27 & 0,98 & 415,92 & 41,65 & 2,49 & 1,00 & 0,99 \\
\hline Diesel $^{\mathrm{b}}$ & $\begin{array}{c}\text { Romanielo } \\
\text { (2011) }\end{array}$ & 136,01 & 4,35 & 0,89 & 339,10 & 8,48 & 2,90 & 2,94 & 0,91 \\
\hline Gasolina & Brandão et al. & 3,71 & 21,10 & 0,97 & 63,40 & 0,27 & 2,60 & 120,48 & 0,99 \\
\hline n-Heptano & $(2010)$ & 317,41 & 2,85 & 0,88 & 363,63 & 284,36 & 1,28 & 1,58 & 0,90 \\
\hline Octano & Romanielo et al. & 83,06 & 19,26 & 0,99 & 414,27 & 51,41 & 6,02 & 13,47 & 1,00 \\
\hline Duodecano & (2008) & 42,33 & 12,87 & 0,97 & 42,32 & 42,33 & 2,84 & 9,98 & 0,98 \\
\hline
\end{tabular}

Fonte: Autoria própria (2017)

Figura 2 - Equilíbrio de adsorção de duodecano e diesel em bagaço de cana

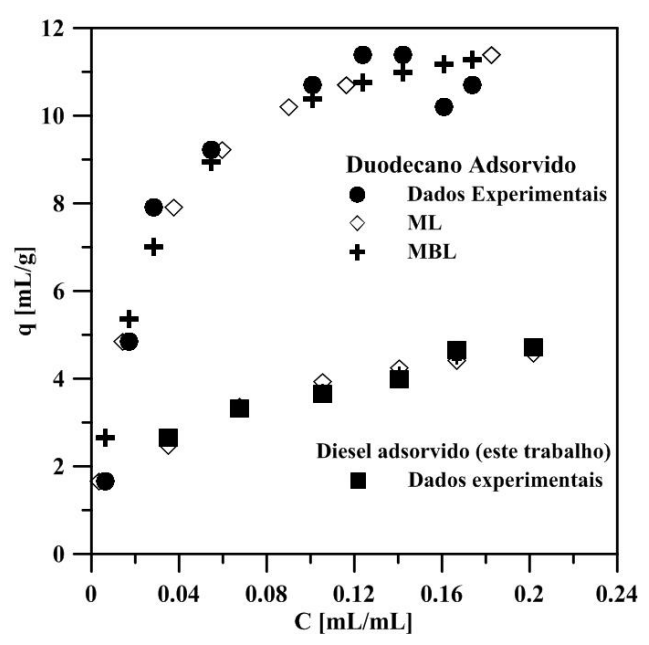




\section{CONCLUSÕES}

O pré-tratamento realizado provavelmente modifica a estrutura do bagaço de cana de modo a obter capacidade adsortivas de diesel similares às obtidas pelo adsorvente tratado sob pressão.

A cinética para adsorção de diesel, utilizando o material em questão é do tipo de segunda ordem.

Os modelos de Langmuir e bi-Langmuir representam quantitativamente a adsorção de derivados do petróleo de soluções aquosas utilizando bagaço de cana como adsorvente.

\section{REFERÊNCIAS}

BAJPAI, A. K.; RAJPOOT, M. Adsorption techniques. Journal of Scientific \& Industrial Research. v. 58, p. 844-860, 1999.

BRANDÃO, P. C., SOUZA T. C., FERREIRA, C. A., HORI, C. E.; ROMANIELO, L. L. Removal of petroleum hydrocarbons from aqueous solution using sugarcane bagasse as adsorbent. Journal of Hazardous Materials. v. 175, n.1-3, p. 1106-1112, 2010.

CHEN, Z., MA, W., HAN, M. Biosorption of nickel and copper onto treated alga (Undaria pinnatifida): Application of isotherm and kinetic models. Journal of Hazardous Materials. v. 155, n. 2, p. 327-333, 2008.

EL-GENDY, N. S., NASSAR, H. N. Study on the effectiveness of spent waste sugarcane for adsorption of different petroleum hydrocarbons water. Desalinization and water treatment. v.5, n. 4, p. 1-15, 2015.

ILJINA, A.; BALTAKYS, K.; EISINAS, A. Gyrolite Adsorption of Zn2+ Ions in Acidic and Alkaline Solutions. Materials Science (Medžiagotyra). v. 21, n. 1, p. 20-25, 2015.

MACRELLI, S.; MOGENSEN, J.; ZACCHI, G. Techno-economic evaluation of 2nd generation bioethanol production from sugar can bagasse and leaves integrated with sugarbased ethanol process. Biotechnology for Biofuels, v. 5, p. 22, 2012.

QIU, H., LV, L., PAN, B., ZHANG, Q., ZHANG, W., ZHANG, Q. Critical Review in Adsorption Kinetic Models. Journal of Zhejiang University Science A, v. 50, p. 716 - 724, 2009.

RODRIGUES, R. F., TREVENZOLI, R. L., SANTOS, L. R. G., LEÃO, V. S.; BOTARO, V. R. Adsorção de metais pesados em serragem de madeira tratada com ácido cítrico. Engenharia Sanitária e Ambiental. v. 11, n. 1, p. 21-35, 2006.

ROMANIELO, L. L., MARQUES, M. G. P, SILVA, F. L. Adsorção de Hidrocarbonetos em Soluções Aquosas Utilizando Bagaço de Cana. In: Anais do $7^{\circ}$ encontro brasileiro sobre adsorção, Campina Grande, 2008.

ROWELL, R. M., KEANY, F. M. Fiberboards made from acetylated bagasse fiber. Wood Fiber Science. v. 23, n. 1, p. 15-22, 1991.

RUBIO, J; RIBEIRO, T. H.; SMITH, R. W. A Dryed Hydrophobic Aquaphyte as an Oil Filter for Oil/Water Emulsions. Spill Science \& Technology Bulletin. v. 8, n. 5-6, p.483-489, 2003

VASCONCELOS, C. B., ROMANIELO, L. L. Adsorção de diesel em bagaço de cana: um estudo teórico experimental da cinética e equilíbrio. In: ANAIS do $6^{\circ}$ Congresso Brasileiro De Termodinâmica aplicada, Salvador, 2011. 